\title{
Variação estacional de Bovicola caprae parasitando caprinos no Estado da Paraíba ${ }^{1}$
}

\author{
Sandra B. Santos ${ }^{2}$, João Luiz H. Faccini ${ }^{3 *}$ e Ana Clara G. Santos ${ }^{4}$
}

\begin{abstract}
Santos S.B., Faccini J.L.H. \& Santos A.C.G. 2006. [Seasonal variation of Bovicola caprae parasitizing goats in the State of Paraíba, northeastern Brazil.] Variação estacional de Bovicola caprae parasitando caprinos no Estado da Paraíba. Pesquisa Veterinária Brasileira 26(4):249253. Departamento de Parasitologia Animal, Instituto de Veterinária, Universidade Federal Rural do Rio de Janeiro, Seropédica, RJ 23890-000, Brazil. E-mail: faccini@ufrrj.br

The effects of temperature, humidity and rainfall on field population trend of the biting louse Bovicola caprae, parasitizing goats bred in the semiarid region of the State of Paraiba, northeastern Brazil were monthly evaluated from August 1999 to July 2002. In the first two years (August 1999 to July 2001) 30 goats were examined, whereas 16 goats were examined in the last year (August 2001 to July 2002). All goats were crossbred, both sexes and abated for human consumption. The lice were collected in an area $4 \mathrm{~cm}$ of diameter at three body sites: maxillary, back line and haunch. Spearman correlation analysis was conducted $(\mathrm{p}<0.05)$ between monthly average intensity of infestation of all stages (egg, larva, nymph, male and female) of the life cycle pooled and monthly total counts of each stage and mean temperature, mean relative humidity and rainfall. B. caprae was found throughout the year with prevalence of $75-100 \%$. Increase of the louse population during the drier months was probably a consequence of poor nutrition due to reduced pasture and inadequate husbandry practices instead the influence of abiotic factors.
\end{abstract}

INDEX TERMS: Bovicola caprae, Trichodectidae, Mallophaga, seasonal variation, goats, northeastern Brazil.

RESUMO.- Os efeitos da temperatura, umidade relativa e precipitação pluvial na população de Bovicola caprae parasitando caprinos na região semi-árida do Estado da Paraíba foram avaliados de agosto 1999 a julho de 2002. Nos dois primeiros anos (agosto de 1999 a julho de 2001) 30 caprinos foram examinados, enquanto que 16 caprinos foram examinados no terceiro ano (agosto de 2001 a julho de 2002). Os animais eram mestiços adultos de ambos os sexos e foram abatidos para consumo humano no Matadouro Público de Patos, PB. As amostras de piolhos foram coletadas de três regiões anatômicas do corpo: maxilar, dorso e glúteo. $O$ teste de Spearman $(\mathrm{p}<0.05)$ foi utilizado na tentativa de se estabelecer uma correlação entre a intensidade média de infestação de todos os estágios e o número total de

\footnotetext{
${ }^{1}$ Recebido em 14 de março de 2006.

Aceito para publicação em 27 de março de 2006.

2 Doutoranda do Curso de Pós-Graduação em Ciências Veterinárias, Universidade Federal Rural do Rio de Janeiro (UFRRJ), Seropédica, RJ 23890000 .

${ }^{3}$ Departamento de Parasitologia Animal, Instituto de Veterinária, UFRRJ, Seropédica, RJ 23890-000. Bolsista do CNPq. *Autor para correspondência: faccini@ufrrj.br

${ }^{4}$ Depto Patologia, Universidade Estadual do Maranhão, São Luiz, MA 65055-970.
}

cada estágio com os fatores abióticos. A prevalência variou entre 75 e 100\% durante o período de estudo. O aumento da população de piolhos nos períodos de seca deveu-se provavelmente a nutrição deficiente dos animais em conseqüência da escassez de pastagem e manejo inadequado do rebanho em vez dos efeitos dos fatores abióticos.

TERMOS DE INDEXAÇÃO: Bovicola caprae, Mallophaga, Trichodectidae, variação estacional, caprinos, Nordeste.

\section{INTRODUÇÃO}

A pediculose dos ruminantes é uma doença parasitária causada por várias espécies de piolhos, tanto hematófagos (Anoplura) como mastigadores (Mallophaga). A diversidade e a distribuição geográfica destes ectoparasitos estão limitadas à presença dos hospedeiros, já que são parasitos obrigatórios permanentes, altamente específicos e sem fase de vida livre no ciclo biológico (Price \& Graham 1997). Neste cenário, a transmissão se processa por contato direto. O parasitismo contínuo pode resultar em irritação,transmissão de agentes patogênicos, além de perdas na produção e produtividade de bovinos, ovinos e caprinos. A amplitude destas perdas depende da espécie e da intensidade de infestação. (Matthysse 1944, Milness et al. 2003). As principais espécies de piolhos que acometem os caprinos domésticos são 
Linognathus stenopsis, Linognathus africanus, Bovicola caprae (Guimarães et al. 2001) e Damalinia limbata (Fourie et al. 1995).

A espécie Bovicola caprae parasita os caprinos em várias regiões do mundo (Kettle 1990). Na região nordeste do Brasil, onde se concentra o maior número de rebanhos, a exploração econômica, predominantemente extensiva e com práticas de manejo inadequadas (Torres 1945, Santos \& Faccini 1996), favorece o parasitismo por esta espécie de piolho.

O ciclo sazonal das espécies de piolhos tem sido mais estudado em ovinos (Scott 1952, Murray 19601963 1968, Wilkinson et al. 1982, James et al. 1998) e bovinos (Chalmer \& Charleston 1980, Milnes et al 2003). Em linhas gerais, as infestações aumentam no inverno e primavera e diminuem no verão. Práticas de manejo adotadas como a tosquia que expõem os piolhos a uma maior incidência de radiação solar ou ainda declínio da imunidade, também contribuem para diminuir ou aumentar a intensidade do parasitismo no verão e inverno, respectivamente. No único artigo publicado sobre sazonalidade de $B$. caprae parasitando caprinos, Kumar et al. (1994) verificaram que na Índia o parasitismo por esta espécie apresenta correlação negativa com temperatura ambiente e fotoperíodo; as intensidades médias de B. caprae são altas durante os meses de inverno, seguidas dos meses de verão e os animais mais fracos são os mais parasitados. No nordeste do Brasil, observações circunstanciais indicam que as infestações por B. caprae se intensificam à medida que progridem os meses secos, o que também pode ser observado para outras ectoparasitoses como a otite parasitária por Psoroptes cuniculi (Costa \& Vieira 1984). Recentemente, Brito et al. (2005) concluíram que nos meses chuvosos (dezembro-abril) o parasitismo dos caprinos por Bovicola caprae, Boophilus microplus, Cochliomyia hominivorax e Dermatobia hominis aumenta em relação aos meses secos (maio-novembro), no Estado do Maranhão. No entanto, esta afirmação baseou-se na análise conjunta das quatro espécies, o que pode não representar a realidade devido à variação interespecífica. $\mathrm{O}$ objetivo deste estudo foi avaliar mais detalhadamente a dinâmica de $B$. caprae em caprinos da mesorregião do sertão paraibano no período de agosto 1999 a julho de 2002 para a elaboração de programas de controle adequados.

\section{MATERIAL E MÉTODOS}

As coletas de Bovicola caprae foram realizadas mensalmente no Matadouro Público de Patos, $\mathrm{PB}$, no período compreendido entre agosto de 1999 e julho de 2002. Nos dois primeiros anos foram examinados 30 animais/mês, totalizando 720 caprinos, enquanto que no terceiro ano foram examinados 16 animais/mês, totalizando 192 caprinos. Os animais utilizados foram caprinos mestiços de ambos os sexos, idade e cor da pelagem variadas, provenientes dos municípios de Patos, São Mamede, Quixaba e São José Bom Fim, localizados na região semi-árida do Estado da Paraíba.

Antes das coletas, os caprinos foram examinados através da inspeção visual para diagnosticar possíveis sinais e/ou lesões na pele, como alopecia, descamação, crostas e nódulos, que pudessem ser associadas com a presença dos malófagos e/ou outros ectoparasitos.

As amostras foram obtidas de três regiões anatômicas do corpo dos caprinos: maxilar, linha dorsal e glúteos. Todos os estágios do ciclo biológico (ovos, ninfas e adultos) foram coletados através do corte do pêlo rente à pele com tesoura e posterior raspagem da mesma com lâmina de bisturi $\mathrm{n}^{\circ} 24$. A escolha das regiões anatômicas foi baseada em observações anteriores publicadas por Santos \& Faccini (1996) na mesma região geográfica, os quais observaram serem estas as regiões mais parasitadas em caprinos. Em cada uma das regiões anatômicas eleitas, foi delimitada uma área de $4 \mathrm{~cm}$ de diâmetro através de cartão de papelão perfurado no centro. Este espaço foi escolhido para evitar danos estéticos na pele e pêlo dos animais, uma vez que após abate dos animais estas peças são comercializadas, e também pelo fato da impraticabilidade de contar o número real de malófagos em toda a região do corpo de cada animal. Metodologia semelhante para contagem de malófagos em caprinos na África do Sul foi utilizada por Fourie et al. (1995), sendo que os autores utilizaram uma área de $5 \mathrm{~cm}$ de diâmetro em seis regiões anatômicas do corpo dos animais para testar a eficácia de um produto à base de diflubenzuron no controle de Damalinia limbata.

Todo o material coletado foi acondicionado em frascos de vidro contendo álcool etílico a 70\% e transportado para o Laboratório de Parasitologia Veterinária do Centro de Saúde e Tecnologia Rural (CSTR), Universidade Federal de Campina Grande, Campus Patos, $\mathrm{PB}$, onde se realizou a contagem e identificação dos estágios presentes (ovos, ninfas e adultos) ao microscópio estereoscópico. Em seguida os malófagos foram clarificados em hidróxido de potássio $(\mathrm{KOH})$ a $10 \%$ a quente, passagem rápida no fenol puro e logo após no Creosoto de Faya e montagem entre lâmina e lamínula em Bálsamo de Canadá, pelo método de Costa Lima (Hoffmann 1987). A identificação dos malófagos foi baseada em Tuff (1977) e Price \& Graham (1997).

Foram obtidos os dados meteorológicos mensais locais de temperatura máxima e mínima $\left(\mathrm{T}^{\circ} \mathrm{C}\right.$ máxima e $\mathrm{T}^{\circ} \mathrm{C}$ mínima), precipitação pluvial $\left(\mathrm{PPmm}^{3}\right)$ e umidade relativa do ar (UR\%), através da estação convencional do $3^{\circ}$ Distrito de Meteorologia (INMET-3 ${ }^{\circ}$ DISME, Recife, PE) do Instituto Nacional de Meteorologia localizado na cidade de Patos, PB (Código OMM:82791).

Para verificar se houve correlação entre o parasitismo por B. caprae e os fatores abióticos locais (temperaturas máxima e mínima, precipitação pluvial e umidade relativa do ar), foi utilizado o teste não paramétrico de correlação de Spearman do programa estatístico Instat $($ (1990-1994 Graphpad software v2.05a).

\section{RESULTADOS}

A pediculose por Bovicola caprae nos caprinos da mesorregião do sertão paraibano foi observada em todos os meses, durante os três anos do estudo. Neste período a prevalência variou entre 75 e 100\% (Quadro1), embora os resultados dos testes estatísticos não tenham apontado nenhuma correlação significativa com os fatores abióticos avaliados quando se analisou a intensidade média de infestação da população total (Quadro 2).

O Quadro 3 contém os dados mensais de $B$. caprae para todas os estágios do ciclo biológico. Verificou que os estágios mais freqüentes em todos os meses foram os ovos e as ninfas indicando a ocorrência de reprodução durante todos os meses do ano. A semelhança com o observado para a intensidade média da população total, não houve correlação significativa com os fatores abióticos avaliados para os estágios de ovo, macho e fêmea. Somente as ninfas apresentaram correlação positiva significativa com a temperatura máxima $\left(r_{s}=0,370\right.$ $\mathrm{p}=0,026 \mathrm{~s}$ ) e correlação negativa significativa com a umidade relativa do ar $\left(r_{s}=-0,332 p=0,047 s\right)$. No entanto, os coeficientes de correlação foram bastante baixos. Os resultados da aná- 
Quadro1. Prevalência e intensidade média de infestação de todos os estágios (ovo, ninfa e adultos) de Bovicola caprae parasitando caprinos na mesorregião do sertão Paraibano e abatidos no Matadouro Público de Patos, PB, entre agosto de 1999 e julho de 2002

\begin{tabular}{|c|c|c|c|c|}
\hline Mês / ano & $\begin{array}{l}\text { Animais } \\
\text { positivos }^{\text {a }}\end{array}$ & $\begin{array}{l}\text { Prevalên- } \\
\text { cia }(\%)\end{array}$ & $\begin{array}{l}\text { Intensidade média } \\
\text { infestação de }\end{array}$ & $\begin{array}{l}\text { Total de pio- } \\
\text { lhos coletados }\end{array}$ \\
\hline Agosto / 1999 & 30 & 100 & $24,8 \pm 7,3$ & 743 \\
\hline Setembro & 28 & 93,3 & $27,4 \pm 10,2$ & 768 \\
\hline Outubro & 27 & 90 & $22,7 \pm 4,9$ & 613 \\
\hline Novembro & 27 & 90 & $30,7 \pm 9,8$ & 829 \\
\hline Dezembro & 28 & 93,3 & $19,0 \pm 5,1$ & 534 \\
\hline Janeiro / 2000 & 29 & 96,7 & $29,2 \pm 11,5$ & 846 \\
\hline Fevereiro & 29 & 96,7 & $25,3 \pm 8,1$ & 733 \\
\hline Março & 30 & 100 & $15,2 \pm 3,7$ & 493 \\
\hline Abril & 28 & 93,3 & $35,5 \pm 10,9$ & 994 \\
\hline Maio & 29 & 96,7 & $31,9 \pm 10,1$ & 924 \\
\hline Junho & 28 & 93,3 & $25,1 \pm 7,2$ & 705 \\
\hline Julho & 29 & 96,7 & $21,1 \pm 8,0$ & 612 \\
\hline Agosto & 30 & 100 & $79,6 \pm 27,5$ & 2388 \\
\hline Setembro & 28 & 93,3 & $26,0 \pm 6,3$ & 729 \\
\hline Outubro & 27 & 90 & $30,8 \pm 8,5$ & 831 \\
\hline Novembro & 27 & 90 & $45,3 \pm 15,6$ & 1224 \\
\hline Dezembro & 25 & 83,3 & $13,5 \pm 4,5$ & 338 \\
\hline Janeiro / 2001 & 29 & 96,7 & $21,1 \pm 5,4$ & 614 \\
\hline Fevereiro & 29 & 96,7 & $22,5 \pm 7,8$ & 652 \\
\hline Março & 27 & 90 & $27,2 \pm 7,2$ & 735 \\
\hline Abril & 29 & 96,7 & $23,1 \pm 8,2$ & 669 \\
\hline Maio & 29 & 96,7 & $30,8 \pm 11,9$ & 894 \\
\hline Junho & 29 & 96,7 & $23,2 \pm 7,8$ & 673 \\
\hline Julho & 30 & 100 & $22,3 \pm 6,7$ & 669 \\
\hline Agosto & 15 & 93,7 & $20,5 \pm 5,1$ & 308 \\
\hline Setembro & 12 & 75 & $17,1 \pm 3,4$ & 205 \\
\hline Outubro & 15 & 93,7 & $22,9 \pm 5,6$ & 344 \\
\hline Novembro & 16 & 100 & $12,6 \pm 2,8$ & 202 \\
\hline Dezembro & 14 & 87,5 & $65,2 \pm 21,9$ & 913 \\
\hline Janeiro / 2002 & 15 & 93,7 & $13,7 \pm 2,0$ & 206 \\
\hline Fevereiro & 15 & 93,7 & $25,6 \pm 9,6$ & 384 \\
\hline Março & 15 & 93,7 & $26,3 \pm 8,4$ & 394 \\
\hline Abril & 14 & 87,5 & $14,7 \pm 4,5$ & 206 \\
\hline Maio & 16 & 100 & $23,1 \pm 8,5$ & 369 \\
\hline Junho & 15 & 93,7 & $26 \pm 7,8$ & 390 \\
\hline Julho & 16 & 100 & $15,1 \pm 4,3$ & 242 \\
\hline Total & 859 & 94,2 & & 23373 \\
\hline
\end{tabular}

a Nos dois primeiros anos foram examinados 30 caprinos/mês e no terceiro ano 16 caprinos/mês.

lise estatística referente aos diferentes estágios do ciclo biológico estão no Quadro 4 e a variação estacional da ninfas na Figura 1.

Os dados meteorológicos obtidos durante os três anos de estudo na mesorregião do sertão paraibano (Quadro 5) registraram as temperaturas mais altas nos meses de outubro de 2000, novembro de 2001, novembro de 2000 e dezembro de 2001 $\operatorname{com} 36,7^{\circ} \mathrm{C}, 36,7^{\circ} \mathrm{C}, 36,2^{\circ} \mathrm{C}$ e $36,1^{\circ} \mathrm{C}$, respectivamente. As temperaturas mais baixas registradas foram em junho de 2002 $\left(20,5^{\circ} \mathrm{C}\right)$, agosto de $1999\left(20,7^{\circ} \mathrm{C}\right)$ e agosto de $2001\left(20,8^{\circ} \mathrm{C}\right)$. Os dados de umidade relativa do ar (\%UR) registraram as mais altas umidades nos meses de janeiro, abril e maio de $2002 \mathrm{com} 72,70$ e $69 \%$, respectivamente, seguidos dos meses de abril de 2000 e março de 2002, ambos com 68\%UR.

Em relação à precipitação pluvial local foram registradas precipitações muito baixas, sendo que na mesorregião do ser-
Quadro 2. Resultado dos Coeficientes de correlação de Spearman entre intensidade média de infestação da população total de Bovicola caprae parasitando caprinos na mesorregião do sertão paraibano entre agosto de 1999 e julho de 2002, e os fatores abióticos

\begin{tabular}{|c|c|c|}
\hline Fatores abióticos & $\begin{array}{l}\text { Coeficiente de } \\
\text { Spearman }\end{array}$ & Teste $\mathrm{t}$ \\
\hline Temperatura máxima ( $\left.{ }^{\circ}{ }^{\circ} \mathrm{Cmín}.\right)$ & $r_{s}=0,126$ & $\mathrm{P}=0,463 n s^{\mathrm{a}}$ \\
\hline Temperatura mínima ( ${ }^{\circ} \mathrm{C}$ máx.) & $\mathrm{r}_{\mathrm{s}}=0,121$ & $\mathrm{P}=0,480 \mathrm{~ns}$ \\
\hline Precipitação pluvial (PPmm³) & $r_{s}=-0,021$ & $\mathrm{P}=0,902 \mathrm{~ns}$ \\
\hline Umidade relativa (UR\%) & $r_{s}=-0,013$ & $\mathrm{P}=0,940 \mathrm{~ns}$ \\
\hline
\end{tabular}

Quadro 3. Número e freqüência dos estágios do ciclo biológico de Bovicola caprae parasitando caprinos na mesorregião do sertão Paraibano entre agosto de 1999 e julho de 2002

\begin{tabular}{|c|c|c|c|c|c|c|c|c|c|}
\hline \multirow[t]{2}{*}{ Mês/ano } & \multicolumn{2}{|c|}{ Fêmeas } & \multicolumn{2}{|c|}{ Machos } & \multicolumn{2}{|c|}{ Ninfas } & \multicolumn{2}{|c|}{ Ovos } & \multirow{2}{*}{$\begin{array}{l}\text { Total } \\
\text { (№) }\end{array}$} \\
\hline & (№) & (\%) & (№) & $(\%)$ & (№) & $(\%)$ & (№) & $(\%)$ & \\
\hline Agosto/1999 & 70 & 9,4 & 38 & 5,1 & 127 & 17,0 & 508 & 68,4 & 743 \\
\hline Setembro & 29 & 3,8 & 19 & 2,5 & 103 & 13,4 & 617 & 80,3 & 768 \\
\hline Outubro & 45 & 7,3 & 38 & 6,1 & 233 & 38,0 & 297 & 48,5 & 613 \\
\hline Novembro & 25 & 3,0 & 20 & 2,4 & 198 & 23,9 & 586 & 70,7 & 829 \\
\hline Dezembro & 24 & 4,5 & 12 & 2,2 & 181 & 33,9 & 317 & 59,4 & 534 \\
\hline Janeiro/2000 & 37 & 4,4 & 22 & 2,6 & 77 & 9,1 & 710 & 83,9 & 846 \\
\hline Fevereiro & 34 & 4,6 & 16 & 2,1 & 157 & 21,4 & 526 & 71,8 & 733 \\
\hline Março & 48 & 9,7 & 33 & 6,7 & 139 & 28,2 & 273 & 55,4 & 493 \\
\hline Abril & 69 & 6,9 & 43 & 4,3 & 185 & 18,6 & 697 & 70,1 & 994 \\
\hline Maio & 53 & 5,7 & 34 & 3,7 & 176 & 19,0 & 661 & 771,5 & 924 \\
\hline Junho & 36 & 5,1 & 18 & 2,6 & 195 & 27,7 & 456 & 64,7 & 705 \\
\hline Julho & 28 & 4,6 & 9 & 1,5 & 73 & 11,9 & 502 & 82,0 & 612 \\
\hline Agosto & 171 & 7,2 & 75 & 3,1 & 317 & 13,3 & 1825 & 76,4 & 2388 \\
\hline Setembro & 50 & 6,8 & 30 & 4,1 & 244 & 33,5 & 405 & 55,6 & 729 \\
\hline Outubro & 34 & 4,0 & 19 & 2,3 & 272 & 32,7 & 506 & 60,9 & 831 \\
\hline Novembro & 59 & 4,8 & 30 & 2,5 & 207 & 16,9 & 928 & 75,8 & 1224 \\
\hline Dezembro & 20 & 5,9 & 3 & 0,9 & 65 & 19,2 & 250 & 73,9 & 338 \\
\hline Janeiro/2001 & 60 & 9,8 & 34 & 5,5 & 141 & 22,9 & 379 & 61,7 & 614 \\
\hline Fevereiro & 24 & 3,7 & 16 & 2,5 & 114 & 17,5 & 498 & 776,4 & 652 \\
\hline Março & 52 & 7,0 & 30 & 4,0 & 196 & 26,7 & 457 & 62,2 & 735 \\
\hline Abril & 55 & 8,2 & 18 & 2,7 & 71 & 10,6 & 525 & 78,5 & 669 \\
\hline Maio & 27 & 3,0 & 20 & 2,2 & 106 & 11,8 & 741 & 82,9 & 894 \\
\hline Junho & 33 & 4,9 & 24 & 3,6 & 116 & 17,2 & 500 & 74,3 & 673 \\
\hline Julho & 42 & 6,2 & 33 & 4,9 & 135 & 20,1 & 459 & 68,6 & 669 \\
\hline Agosto & 30 & 9,7 & 14 & 4,5 & 78 & 25,3 & 186 & 60,4 & 308 \\
\hline Setembro & 22 & 10,7 & 12 & 55,8 & 97 & 47,3 & 74 & 36,0 & 205 \\
\hline Outubro & 36 & 10,5 & 8 & 2,3 & 103 & 29,9 & 197 & 57,3 & 344 \\
\hline Novembro & 13 & 6,4 & 11 & 5,4 & 88 & 43,6 & 90 & 44,6 & 202 \\
\hline Dezembro & 31 & 3,4 & 21 & 2,3 & 188 & 20,6 & 673 & 73,7 & 913 \\
\hline Janeiro/2002 & 37 & 17,9 & 16 & 7,8 & 79 & 38,3 & 74 & 35,9 & 206 \\
\hline Fevereiro & 25 & 6,5 & 6 & 1,6 & 42 & 10,9 & 311 & 80,9 & 384 \\
\hline Março & 26 & 6,6 & 19 & 4,8 & 64 & 16,2 & 285 & 72,3 & 394 \\
\hline Abril & 16 & 7,8 & 3 & 1,5 & 45 & 21,8 & 142 & 68,9 & 206 \\
\hline Maio & 29 & 7,9 & 6 & 1,6 & 38 & 10,3 & 296 & 80,2 & 369 \\
\hline Junho & 33 & 8,5 & 15 & 3,8 & 73 & 18,7 & 269 & 68,9 & 390 \\
\hline Julho & 13 & 5,3 & 9 & 3,7 & 62 & 25,6 & 158 & 65,3 & 242 \\
\hline
\end{tabular}

tão paraibano, durante maior parte do ano, predominam os meses "secos" com chuvas escassas ou inexistentes, tendo apenas duas estações definidas: período seco e chuvoso, e mesmos nas estações chuvosas a precipitação média não ultrapassa $660 \mathrm{~mm}^{3}$, característica das regiões semi-áridas do nordeste brasileiro. Neste período as precipitações mais altas registradas foram de 380,4, 223,4 e 156,0 $\mathrm{mm}$ nos meses de janeiro e março 
de 2002 , março de 2000 respectivamente. As mais baixas precipitações foram de $0,2,0,3$ e 1,2mm para os meses de novembro de 2001, julho de 2002 e novembro de 2000. Nos meses de

Quadro 4. Resultado dos Coeficientes de Spearman entre número de fêmeas, machos, ninfas e ovo da população total de

Bovicola caprae parasitando caprinos procedentes da mesorregião do sertão paraibano entre agosto de 1999 e julho de 2002, e os fatores abióticos

\begin{tabular}{lcccc}
\hline Estágios & \multicolumn{4}{c}{ Fatores abióticos } \\
\cline { 2 - 5 } Fêmeas & $\mathrm{T}^{\circ} \mathrm{C}$ máxima & $\mathrm{T}^{\circ} \mathrm{C}$ mínima. & $\mathrm{PP} \mathrm{mm}$ & $\mathrm{UR} \%$ \\
& $\mathrm{r}=0,181$ & $\mathrm{r}_{\mathrm{s}}=-0,142$ & $\mathrm{r}_{\mathrm{s}}=0,115$ & $\mathrm{r}_{\mathrm{s}}=-0,332$ \\
\multirow{3}{*}{ Machos } & $\mathrm{r}_{\mathrm{s}}=0,013$ & $\mathrm{r}_{\mathrm{s}}=-0,051$ & $\mathrm{r}_{\mathrm{s}}=-0,012$ & $\mathrm{r}_{\mathrm{s}}=-0,145$ \\
& $\mathrm{p}=0,936 \mathrm{~ns}$ & $\mathrm{p}=0,766 \mathrm{~ns}$ & $\mathrm{p}=0,945 \mathrm{~ns}$ & $\mathrm{p}=0,396 \mathrm{~ns}$ \\
Ninfas & $\mathrm{r}_{\mathrm{s}}=0,370$ & $\mathrm{r}_{\mathrm{s}}=0,276$ & $\mathrm{r}_{\mathrm{s}}=-0,180$ & $\mathrm{r}_{\mathrm{s}}=-0,332$ \\
& $\mathrm{p}=0,026 \mathrm{~s}$ & $\mathrm{p}=0,102 \mathrm{~ns}$ & $\mathrm{p}=0,292 \mathrm{~ns}$ & $\mathrm{p}=0,047 \mathrm{~s}$ \\
Ovos & $\mathrm{r}_{\mathrm{s}}=0,117$ & $\mathrm{r}_{\mathrm{s}}=0,150$ & $\mathrm{r}_{\mathrm{s}}=-0,069$ & $\mathrm{r}_{\mathrm{s}}=-0,136$ \\
& $\mathrm{p}=0,495 \mathrm{~ns}$ & $\mathrm{p}=0,379 \mathrm{~ns}$ & $\mathrm{p}=0,685 \mathrm{~ns}$ & $\mathrm{p}=0,428 \mathrm{~ns}$
\end{tabular}

$\overline{\mathrm{a}} \mathrm{ns}=$ não significativo,, $\mathrm{s}=$ significativo.

Quadro 5. Distribuição mensal das médias de temperatura máxima e mínima, umidade relativa do ar e precipitação pluvial entre agosto de 1999 e julho de $2002^{\mathrm{a}}$

\begin{tabular}{|c|c|c|c|c|}
\hline \multirow[t]{2}{*}{ Mês/ano } & \multicolumn{2}{|c|}{ Temperatura $\quad\left({ }^{\circ} \mathrm{C}\right)$} & \multirow{2}{*}{$\begin{array}{c}\text { Umidade } \\
\text { Relativa (\%) }\end{array}$} & \multirow{2}{*}{$\begin{array}{l}\text { Precipitação } \\
\text { Pluvial }\left(\mathrm{mm}^{3}\right)\end{array}$} \\
\hline & Máxima & Mínima & & \\
\hline Agosto/1999 & 33,3 & 20,7 & 48 & 0 \\
\hline Setembro & 35 & 22,4 & 52 & 0 \\
\hline Outubro & 35 & 22,6 & 45 & 19,4 \\
\hline Novembro & 35,6 & 23,4 & 46 & 16,7 \\
\hline Dezembro & 34,9 & 23,7 & 52 & 90,4 \\
\hline Janeiro/2000 & 33,2 & 22,9 & 49 & 40,6 \\
\hline Fevereiro & 33,4 & 22,6 & 66 & 151,3 \\
\hline Março & 33,1 & 22,4 & 66 & 156 \\
\hline Abril & 32,6 & 22,4 & 68 & 92 \\
\hline Maio & 33,6 & 21,8 & 57 & 30,9 \\
\hline Junho & 32,9 & 21,4 & 59 & 21,5 \\
\hline Julho & 32,5 & 21,1 & 58 & 9,1 \\
\hline Agosto & 32,6 & 21,9 & 58 & 64,2 \\
\hline Setembro & 34,2 & 22,9 & 55 & 9,7 \\
\hline Outubro & 36,7 & 23,2 & 47 & 0 \\
\hline Novembro & 36,2 & 24 & 45 & 0,3 \\
\hline Dezembro & 34,6 & 22,9 & 50 & 51,7 \\
\hline Janeiro/2001 & 35,7 & 22,4 & 46 & 14,4 \\
\hline Fevereiro & 36,6 & 24,6 & 44 & 7,2 \\
\hline Março & 34,5 & 23,6 & 60 & 171,2 \\
\hline Abril & 32,7 & 23,2 & 64 & 168,7 \\
\hline Maio & 35,2 & 23,3 & 51 & 11,4 \\
\hline Junho & 31,8 & 21,9 & 44 & 81,8 \\
\hline Julho & 31,5 & 21,4 & 58 & 11,2 \\
\hline Agosto & 32,9 & 20,8 & 50 & 0 \\
\hline Setembro & 35 & 22,4 & 47 & 6,2 \\
\hline Outubro & 35,6 & 23,4 & 46 & 2,6 \\
\hline Novembro & 36,7 & 23,9 & 44 & 0,2 \\
\hline Dezembro & 36,1 & 24,2 & 48 & 21,7 \\
\hline Janeiro/2002 & 32,3 & 22,8 & 72 & 380,4 \\
\hline Fevereiro & 33,5 & 22,3 & 62 & 96,9 \\
\hline Março & 32,8 & 22,5 & 68 & 223,4 \\
\hline Abril & 32,6 & 22,6 & 70 & 110,9 \\
\hline Maio & 32,3 & 21,9 & 69 & 129,3 \\
\hline Junho & 31,5 & 20,5 & 67 & 64,8 \\
\hline Julho & 33,4 & 20,9 & 55 & 1,2 \\
\hline
\end{tabular}

a Fonte: Inmet (Instituto Nacional de Meteorologia), Rio de Janeiro.

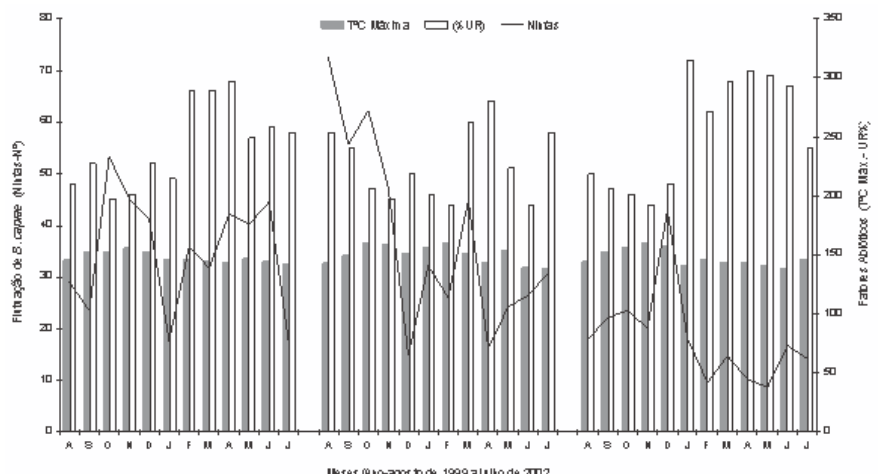

Fig.1. Flutuação de ninfas de Bovicola caprae parasitando caprinos na mesorregião do sertão paraibano entre agosto de 1999 e julho de 2002 e os fatores abióticos temperatura máxima e umidade relativa.

agosto e setembro de 1999, outubro de 2000 e agosto de 2001 a precipitação foi zero, não ocorrendo chuvas na região.

\section{DISCUSSÃO}

A prevalência de Bovicola caprae na região estudada foi semelhante à observada por Costa \& Vieira (1984) no Ceará a qual variou de $62,2-100 \%$, e por Brito et al. (2005) no Maranhão, $80,7 \%$.

O exame clínico dos animais parasitados revelou pêlos sem brilho, grossos e quebradiços.

Em relação à variação estacional, os dados indicam que o estágio ninfal é o mais sensível aos fatores abióticos, principalmente a umidade, diminuindo em quantidade a medida que esta aumenta em conseqüência do aumento das chuvas. No entanto, não foi possível imputar somente à umidade, $o$ aumento ou diminuição da população de piolhos como proposto por Costa e Vieira (1984) para a região de Sobral, no Estado do Ceará, já que o coeficiente de correlação obtido foi muito baixo. Estes autores verificaram que a população de $B$. caprae apresenta picos durante os meses secos e diminuem à medida que se iniciam as chuvas. Ressalta-se que a precipitação média registrada no período de estudo foi de $62,7 \mathrm{~mm}^{3}$, ao contrário das verificadas por Costa \& Vieira (1984), onde as precipitações pluviais médias foram superiores a $300 \mathrm{~mm}^{3}$, demonstrando com isso a variação climática existente entre as duas regiões estudadas, sendo, portanto necessário caracterizar cada região bioclimatologicamente, antes de estabelecer relação com o parasitismo por B. caprae. Pode-se inferir que na mesorregião do sertão Paraibano, o aumento da infestação durante os períodos secos seja também devido à debilidade dos animais, pelo mau estado nutricional resultante da escassez de alimentos comum nesta região. Alie-se a isto as precárias condições de manejo adotadas pelos pequenos criadores, traduzidas pela aglomeração dos animais, independentemente de idade, sexo e estado fisiológico, tal como a prenhez (Costa \& Vieira 1984). Este cenário favorece, sobremaneira, a transmissão dos piolhos. Estudos realizados por Chalmers \& Charleston (1980b) em bovinos mostraram-se semelhantes, onde a aglomeração dos bovinos nos estábulos favorecia o contato direto entre os animais e transmissão de Bovicola bovis e Linognathus vitulli. Watson et al. (1997) também observaram o 
mesmo em bovinos parasitados com $B$. bovis. Ao iniciarem-se as chuvas na mesorregião do sertão paraibano existe uma maior disponibilidade de alimentos e os animais melhoram seu estado nutricional, conseqüientemente há melhora na resistência.

Embora não se tenham dados sobre a biologia de B. caprae em condições naturais, Mathysse (1944) demonstrou experimentalmente que as fêmeas de B. bovis são altamente ativas e prolí-feras, podendo também se reproduzir partenogeneticamente. Estes aspectos da biologia, somados às condições de manejo inadequado dos animais, contribuem para que os malófagos sejam transmitidos com facilidade entre os rebanhos e estabeleçam novas populações em curto período de tempo. $O$ autor também cita que o estágio de ninfa de $B$. bovis é o mais ativo e sofre marcada influência da temperatura ambiente e do corpo do hospedeiro. Ainda segundo Castro (1984), a temperatura do corpo do hospedeiro, bem como pêlos com espessura máxima, seriam responsáveis por um microhabitat ideal para o desenvolvimento de B. caprae.

Estudos realizados por Murray (1957a,b, 1963) na Austrália mostraram que existem vários fatores envolvidos na variação estacional de $B$. ovis, dentre estes, o excesso de precipitações pluviais são prejudiciais para estes malófagos, devido ao encharcamento do velo dos ovinos. Em condições experimentais Murray (1960) observou que umidades de $100 \%$ cessam o desenvolvimento dos ovos, a imersão em água por uma hora é letal para $50 \%$ dos ovos e o velo embebido em água causa morte em $75-100 \%$ de ninfas e adultos. Altas temperaturas são prejudiciais ao desenvolvimento de $B$. ovis dos ovinos e a prática da tosquia realizada no final do período seco expõe os malófagos a radiação solar direta, que é letal para os mesmos, contribuindo para diminuição nas densidades populacionais deste malófago. Mathysse (1944) observou que B. bovis é sensível a atmosferas saturadas de água.

Embora os malógafos sejam parasitos permanentes, Heath (1973) demonstrou que fêmeas de $B$. caprae podem sobreviver e ovipor fora do hospedeiro cerca de 3 dias em temperaturas variando de $25-35^{\circ} \mathrm{C}$ e umidade relativa variando de $10-90 \%$.

0 resultado deste experimento indica claramente que os fatores abióticos investigados não são determinantes na variação sazonal de B. caprae na região estudada.

\section{REFERÊNCIAS}

Brito D.R.B., Santos A.C.G. \& Guerra R.M.S.N. 2005. Ectoparasitos em rebanhos de caprinos e ovinos na microrregião do Alto Mearim e Grajaú, Estado do Maranhão. Revta Bras. Parasitol. Vet. 14(2):59-63

Castro A.1984. A Cabra. $3^{\text {a }}$ ed. Freitas Bastos Editora, Rio de Janeiro, RJ. $372 \mathrm{p}$.

Chalmers K. \& Charleston W.A.G.1980. Cattle lice in New Zealand: observations on the prevalence, distribution and seasonal patterns of infestation. N.Z. Vet. J. 28(10):198-200.

Costa A.F.C. \& Vieira L.S. 1984. Ectoparasitos permanentes de caprinos e ovinos em Sobral-CE. Pesq. Agropec. Bras. 19(5):639-646.

Fourie L.J., Kok D.J., Allan M.J. \& Oberem P.T. 1995. The efficacy of diflubenzuron against the body louse (Damalinia limbata) of angora goats. Vet. Parasitol. 59(3-4):257-262.

Guimarães J.H., Tucci, E.C. \& Barros-Battesti D.M. 2001. Ectoparasitos de Importância Veterinária. Plêiade/FAPESP, São Paulo. 218p.

Heath A.C.G. 1973. The biology and survival of starved cattle and goat biting lice (Mallophaga) at different temperatures and relative humidities. N.Z. Entomol. 5(3-4):330-334.

Hoffmann, R. P.1987. Diagnóstico do Parasitismo Veterinário. Sulina, Porto Alegre, RS. 156p.

IBGE - Instituto Brasileiro de Geografia e Estatística. Acesso em 25 de junho de 2004. http://www.ibge.gov.br

James P.J., Moon R.D. \& Brown D.R. 1998. Seasonal dynamics and variation among sheep in densities of the sheep biting louse, Bovicola ovis. Int. J. Parasitol. 28(2):283-292.

Kettle D.S.1990. Medical and Veterinary Entomology. Wiley \& Sons, New York. 658p.

Kumar A., Rawat B.S., Saxena, A.K. \& Agarwal G.P. 1994. Prevalence of ectoparasites on goats in Dehradun (India). Appl. Parasitol. 35(3):227236.

Matthysse J.G. 1944. Biology of cattle biting louse and notes on cattle sucking lice. J. Econ. Entomol. 37(3):436-442.

Milnes A.S., O'Callaghan C.J. \& Green L.E. 2003. A longitudinal study of a natural lice infestation in growing cattle over two winter periods. Vet. Parasitol. 116(1):67-83.

Murray M.D.1957a. The distribution of the eggs of mammalian lice on their hosts. Aust. J. Zool. 5(2):173-187.

Murray M.D. 1957b. The distribution of eggs of mammalian lice on their hosts. II. Analysis of the oviposition behaviour of Damalinia ovis (L.). Aust. J. Zool. 5(2):19-29.

Murray M.D. 1960. The ecology of lice on sheep II. The influence of temperature and humidity on the development and hatching of the eggs of Damalinia ovis (L.). Aust. J. Zool. 8(3):357-362.

Murray M.D. 1963. The ecology of lice on sheep. V. Influence of heavy rain on populations of Damalinia ovis (L.). Aust. J. Zool. 11(2):173-182.

Murray M.D. 1968. Ecology of lice on sheep. VI. The influence of shearing and solar radiation on populations and transmission of Damalinia ovis, Aust. J. Zool. 16(5):725-738.

Price M.A. \& Graham O.H. 1997. Chewing and sucking lice as parasites of mammals and birds. Tech. Bull. 1849, Agric. Res. Service, US Dept Agriculture. 257p.

Santos A.C.G. \& Faccini J.L.H. 1996. Estudo seccional da piolheira caprina causada por Damalinia caprae (Gurlt, 1843) (Thichodectidae: Mallophaga) na região do semi-árido do Estado da Paraíba. Revta Bras. Parasitol. Vet. $5(1): 43-46$.

Scott M.T. 1952. Observations on the bionomics of the sheep body louse (Damalinia ovis). Aust. J. Agric. Res. 3(1):60-67.

Torres S. 1945. Doenças dos Caprinos e Ovinos no Nordeste Brasileiro. Serv. Inf. Agrícola, Min. Agricultura, Rio de Janeiro, RJ. 34p.

Tuff D.W. 1977. A key to the lice of man and domestic animals. Texas J. Sci. 28(1-4):145-158.

Watson D.W., Lloyd J.E. \& Kumar R. 1997. Density and distribution of cattle lice (Phthiraptera: Haematopinidae, Linognathidae, Trichodectidae) on six steers. Vet. Parasitol. 69(3-4):283-296.

Wilkinson F.C., Chaneet G.C. \& Beetson B.R. 1982. Growth of population of lice, Damalinia ovis, on sheep and their effects on production and processing performance of wool. Vet. Parasitol. 9(3-4):171-177. 